\title{
Power-Minimizing Resource Allocation in Multiuser Cooperative Relay Communications
}

\author{
Bin Chen, Youming Li, Yaohui Wu, Xiaoqing Liu, Ting Zou \\ Institute of Communication Technology, Ningbo University, Ningbo 315211, China \\ Email: liyouming@nbu.edu.cn
}

Received June, 2013

\begin{abstract}
In this paper, we investigate the power-minimizing resource allocation problem in multiuser cooperative relay communication systems. A joint optimization problem involving subcarrier assignment, relay selection and power allocation is formulated. Since the problem cannot be solved directly, we decompose it into three subproblems. According to the equivalent channel gains and the target rates of users, the subcarrier assignment and relay selection are conducted. Motivated by the water-filling algorithm, we propose a power allocation algorithm with cooperative features. Simulations results indicate that the proposed algorithm performs better in terms of the total transmit power consumption than the existing algorithms.
\end{abstract}

Keywords: Subcarrier Assignment; Relay Selection; Power Allocation; Cooperative Features

\section{Introduction}

As the demand for high data-rate multi-media wireless services increases rapidly, the third generation (3G) wireless communication systems have been unable to meet this requirement. Therefore, researchers are working on the future fourth generation (4G) wireless communication systems.

Orthogonal frequency division multiple access (OFDMA) is regarded as a promising technology for the $4 \mathrm{G}$ systems, which can offer high spectral efficiency and mitigate frequency-selective fading. Besides OFDMA, the 4G systems adopt many other key technologies. Cooperative relaying, assisted by additional relay stations, can increase the coverage and obtain spatial diversity. By combining these technologies, resource allocation in OFDMA systems has drawn much attention recently. According to different optimization objectives and constraints, the adaptive resource allocation schemes for OFDMA systems can be roughly divided into two categories: Rate adaptive (RA) schemes to maximize the system throughput [1-3]; and margin adaptive (MA) schemes to minimize the overall transmit power [4-9]. There are many works that investigate the MA schemes. Followed with global warming, the growth in energy consumption provides new topics and issues in communication systems. Hence, how to reduce energy consumption while meeting throughput requirement in such communication systems is an urgent task, which is known as green communication.
In [5], the authors proposed a low-complexity algorithm based on the Lagrange dual decomposition theory to minimize the downlink transmit power in MIMOOFDMA systems. In [6], Lin et al. proposed an algorithm to find suboptimal and optimal solutions to sum power minimization resource allocation problems in OFDMA-based networks. In order to minimize the total transmit power in cooperative uplink systems, the authors in [7] derived two algorithms based on the flow-optimized cooperative scheme (FCS) and the single-relay cooperative scheme (SCS), respectively. Reference [8] considered the problem of energy-efficient resource and power allocation in the uplink of multiuser multichannel OFDM-based systems, and proposed to maximize the energy efficiency (EE). In [9], the authors aimed at minimizing the overall transmit power under total power and target data constraints in cooperative multiuser OFDMA systems and then proposed a threestep iterative suboptimal assignment algorithm.

In this paper, we investigate the power-minimizing resource allocation problem in multiuser cooperative relay communications. We formulate the problem as a joint optimization problem involving subcarrier assignment, relay selection and power allocation. Since the problem cannot be solved directly, we decompose it into three subproblems. Firstly, according to the average channel gains and the target rates of users, we decide the number of subcarriers that users will be assigned. Secondly, we assign users to the subcarriers with the best equivalent channel gains meanwhile selecting relays to 
the users. Finally, combined with cooperative features, we propose a power allocation algorithm based on the water-filling algorithm. Simulations results indicate that the proposed algorithm performs better in terms of the total transmit power consumption than the existing algorithms, while meeting the target rates of users.

The remainder of this paper is organized as follows. Section 2 provides the system model and formulates the resource allocation problem. Section 3 analyzes the optimization problem and proposes the algorithm. Simulation results are given and discussed in Section 4. Finally, Section 5 draws the conclusions.

\section{System Model and Problem Formulation}

In this section, we first describe the model for multiuser cooperative relay communication systems, and then formulate the resource allocation problem.

\subsection{System Model}

We consider an OFDMA-based uplink cooperative relay communication system as shown in Figure 1. There are $K$ mobile stations (MS) and $M$ relay stations (RS) transmitting on $N$ subcarriers to one base station (BS), where all stations are equipped with only one antenna. Due to long distance and heavy blockage, it is assumed that there is no direct transmission between the BS and the MSs. We consider two phases in uplink relay transmission. During the first phase, each MS $k$ broadcasts its data to available RSs. In the second phase, the RSs forward the data to the BS, where we only consider decode-and-forward (DF) mode. For simplicity, we assume that the RSs forward the received data to the BS on the same subcarrier. It is further assumed that the channels are slow fading, thus the channel state information (CSI) of all links can be estimated and fed back to the BS.

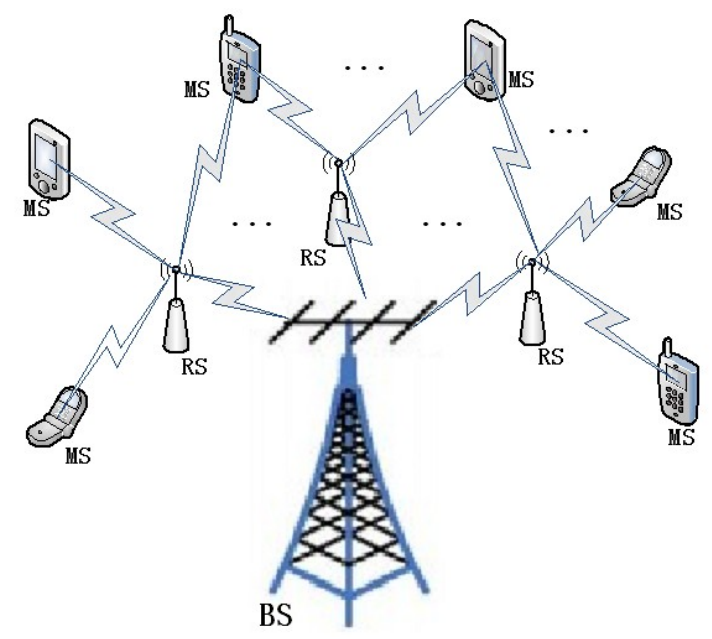

Figure 1. The system model of uplink cooperative relay communication.
Let $H_{k, r, n}$ and $H_{r, B, n}$ represent respectively the channel gains between $k$ th MS and $r$ th RS, $r$ th RS and BS on subcarrier $n$. The transmit powers of $k$ th MS to $r$ th $\mathrm{RS}$, and $r$ th RS to BS spent on subcarrier $n$ are $p_{k, r, n}$ and $p_{r, B, n}$, respectively. According to Shannon formula, the achievable rate of $k$ th MS on subcarrier $n$ forwarded by $r$ th RS is given by

$$
\begin{aligned}
r_{k, n}=\frac{1}{2} \min \left\{\log _{2}\left(1+p_{k, r, n} H_{k, r, n}\right),\right. \\
\left.\log _{2}\left(1+p_{r, B, n} H_{r, B, n}\right)\right\}
\end{aligned}
$$

\subsection{Problem Formulation}

We define $\rho_{k, r, n} \in\{0,1\}$ as the subcarrier assignment variable, where $\rho_{k, r, n}=1$ indicates the assignment of subcarrier $n$ to $k$ th MS and $r$ th RS pair, and $\rho_{k, r, n}=0$, otherwise. Our goal is to minimize the total transmit power while meeting the target rates of users, so that the optimization problem can be formulated as

$$
\begin{aligned}
& \min \sum_{n=1}^{N} \sum_{r=1}^{M} \rho_{k, r, n}\left(\sum_{k=1}^{K} p_{k, r, n}+p_{r, B, n}\right) \\
& \text { s.t. } \text { C1. } \rho_{k, r, n} \in\{0,1\}, \forall k, r, n ; \\
& \quad \text { C2. } \sum_{n=1}^{N} \sum_{r=1}^{M} \rho_{k, r, n}=1, \forall n ; \\
& \text { C3. } p_{k, r, n} \geq 0, p_{k, B, n} \geq 0, \forall k, r, n ; \\
& \quad \text { C4. } \sum_{n=1}^{N} r_{k, n} \geq R_{k}, \forall k, n
\end{aligned}
$$

Here, constraint C2 guarantees that every subcarrier is allocated to at most one RS-MS pair. C3 indicates the powers of MSs and RSs are non-negative. In C4, $R_{k}$ is the target rate of $k$ th MS.

\section{Resource Allocation Algorithm}

The optimization in (2) is a joint optimization problem. Since it cannot be solved directly, we decompose it into three subproblems: the number of subcarrier assignment, subcarrier assignment and relay selection, and power allocation.

\subsection{The Number of Subcarrier Assignment}

In order to maximize (1), we can obtain that

$$
p_{k, r, n} H_{k, r, n}=p_{r, B, n} H_{r, B, n}
$$

Thus the sum power consumed in the link of $k$ th MS on subcarrier $n$ forwarded by $r$ th RS is

$$
p_{k, B, n}=p_{k, r, n}+p_{r, B, n}
$$

According to [3], the equivalent channel gain in the link of $k$ th MS on subcarrier $n$ forwarded by $r$ th RS is 


$$
H_{k, r, n}^{\text {equ }}=\frac{H_{k, r, n} H_{r, B, n}}{H_{k, r, n}+H_{r, B, n}}
$$

Consequently, Equation (1) can be rewritten as

$$
r_{k, n}=\frac{1}{2} \log _{2}\left(1+p_{k, B, n} H_{k, r, n}^{e q u}\right)
$$

Based on [4], we assume that each MS $k$ experiences an average channel gain on every subcarrier with

$$
H_{k}=\frac{\sum_{n=1}^{N} \sum_{r=1}^{M} H_{k, r, n}^{e q u}}{M \cdot N}
$$

Let MS $k$ be allocated $m_{k}$ subcarriers. When the gain on each subcarrier is the same, the optimal rate-power allocation is to transmit $R_{k} / m_{k}$ bits on each subcarrier, resulting in total transmit power as $m_{k}\left(2^{R_{k} / m_{k}}-1\right) / H_{k}$. Thus in order to determine the number of subcarriers assigned to each MS $\left\{m_{k}, k=1,2, \ldots, K\right\}$, the objective function can be expressed as

$$
\begin{aligned}
& \min \sum_{k=1}^{K} \frac{m_{k}}{H_{k}}\left(2^{\frac{R_{k}}{m_{k}}}-1\right) \\
& \text { s.t. C1. } \sum_{k=1}^{K} m_{k}=N, \forall k \\
& \quad \text { C2. } m_{k} \in\left\{\left[\frac{R_{k}}{B_{\max }}\right], \ldots, N\right\}, \forall k
\end{aligned}
$$

where $B_{\max }$ is the number of maximum modulation bit.

\subsection{Subcarrier Assignment and Relay Selection}

According to equivalent channel gain, we should assign the better subcarriers to each MS. Because every subcarrier is allocated to at most one RS-MS pair, we propose an algorithm of subcarriers selecting users. The algorithm is as follows.

a) Find the maximum equivalent channel gain for each MS on each subcarrier.

$$
H_{k, n}=\max _{1 \leq r \leq M} H_{k, r, n}^{e q u}
$$

b) According to the average channel gain of each subcarrier $H_{n}=\sum_{k=1}^{K} H_{k, n} / K$, arrange the channel gain matrix $\mathrm{H}(K, N)$ in increasing order.

$$
\mathrm{H}(K, N),\left(H_{1}<H_{2}<\ldots<H_{N}\right)
$$

c) For each subcarrier, find the MS whose channel gain is the largest on the subcarrier, then assign the subcarrier to the MS. Detailed process is as follows.

$$
\text { for } \begin{aligned}
n=1,2, \ldots, N \quad \text { do } \\
k \leftarrow \arg \max _{1 \leq k \leq K} H_{k, n}
\end{aligned}
$$

$$
\begin{aligned}
\text { while } c_{k} & =m_{k} d o \\
H_{k, n}, & \forall n \leftarrow 0 \\
k & \leftarrow \arg \max _{1 \leq k \leq K} H_{k, n}
\end{aligned}
$$

end while

$$
\begin{aligned}
& \qquad C_{k} \leftarrow C_{k} \cup\{n\} \\
& c_{k} \leftarrow c_{k}+1 \\
& \text { end for }
\end{aligned}
$$

where $C_{k} \subseteq\{1,2, \ldots, N\}$ indicates the set of subcarriers assigned to the $k$ th MS, , $c_{k} \in\{1,2, \ldots, N\}$ is the number of elements in the set $C_{k}$.

\subsection{Power Allocation}

Based on the water-filling algorithm, we propose a power allocation algorithm, which also has cooperative features. The algorithm is as follows.

a) Extract the channel gain of the subcarriers assigned to the MS from the channel gain matrix $\mathrm{H}(K, N)$, and store them into a row vector $\left\{h\left(k, m_{k}\right), k=1,2, \ldots, K\right\}$.

b) Arrange $h\left(k, m_{k}\right)$ in decrease order.

$$
\begin{gathered}
h\left(k, m_{k}\right)=\left[h_{k, 1}, h_{k, 2}, \ldots, h_{k, m_{k}}\right] \\
\left(h_{k, 1}>h_{k, 2}>\ldots>h_{k, m_{k}}\right)
\end{gathered}
$$

c) Compute the water-filling constant.

$$
K_{M A, k}=\left(\frac{2^{R_{k}}}{\prod_{n=1}^{m_{k}} h_{k, n}}\right)^{\frac{1}{m_{k}}}
$$

d) Test subcarrier energy.

$$
\varepsilon_{m_{k}}=K_{M A, k}-1 / h_{k, m_{k}}<0
$$

If yes, then $m_{k} \leftarrow m_{k}-1$, go to step c), otherwise continue.

f) Compute subcarrier energy and rate

$$
\begin{gathered}
\varepsilon_{k, n}=K_{M A, k}-1 / h_{k, n}, n=1,2, \ldots, m_{k} \\
b_{k, n}=\log _{2}\left(K_{M A, k} \cdot h_{k, m_{k}}\right)
\end{gathered}
$$

\section{Simulation Results and Analysis}

In this section, simulation results are provided to evaluate the system performance. Here, six-path frequency-selective Rayleigh fading channels are considered. The maximum Doppler shift is $30 \mathrm{~Hz}$. The total bandwidth is set to be $1 \mathrm{MHz}$ and the number of subcarriers is 256 . Assume that the Gaussian white noise power spectral density is $-36 \mathrm{~dB} / \mathrm{Hz}$ and the number of maximum modulation bit is 4. The total power consumption of each power allocation scheme is averaged over 1,000 independent Monte-Carlo simulations. The performance comparison is conducted among the algorithm in [9], static resource allocation 
algorithms based on Greedy and Water-Filling algorithm, respectively, and the pro- posed algorithm.

Figure 2 indicates the total transmit power consumption in uplink versus the total number of MSs. In order to reflect different wireless services, the target rates of MSs are $1 \sim 10 \mathrm{~b} / \mathrm{s} / \mathrm{Hz}$. It is seen that for a fixed total number of MSs, the total transmit power consumption under the proposed algorithm is always the smallest among the four algorithms. Meanwhile, with the increase of the total number of MSs, the total transmits power consumption under the proposed algorithm increases at the slowest speed.

Figure 3 illustrates the total transmit power consumption in uplink versus the total target rate of $10 \mathrm{MSs}$. In the condition of a fixed total target rate, we conduct equal rate allocation for $10 \mathrm{MSs}$. It is observed that the total transmit power of the proposed algorithm is the lowest among the four algorithms.

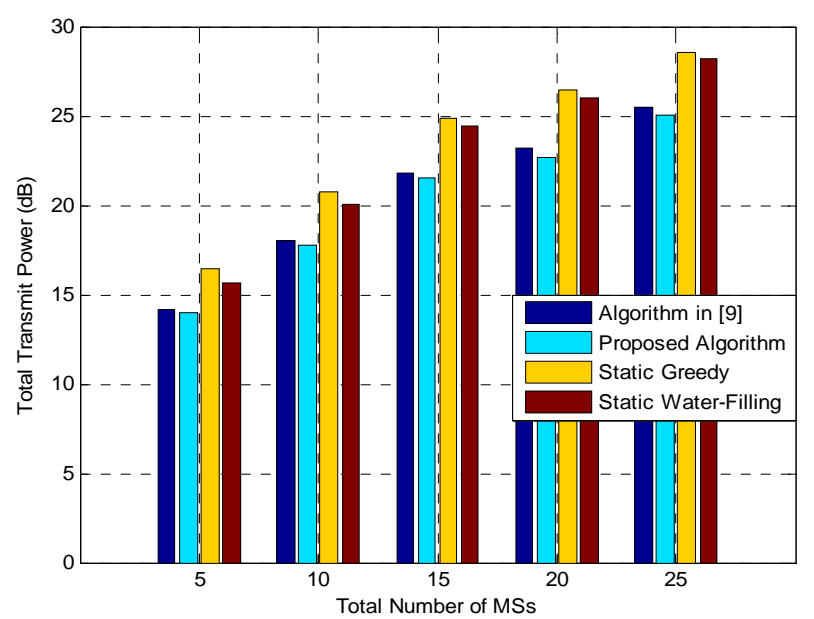

Figure 2. Total transmit power in different number of MSs.

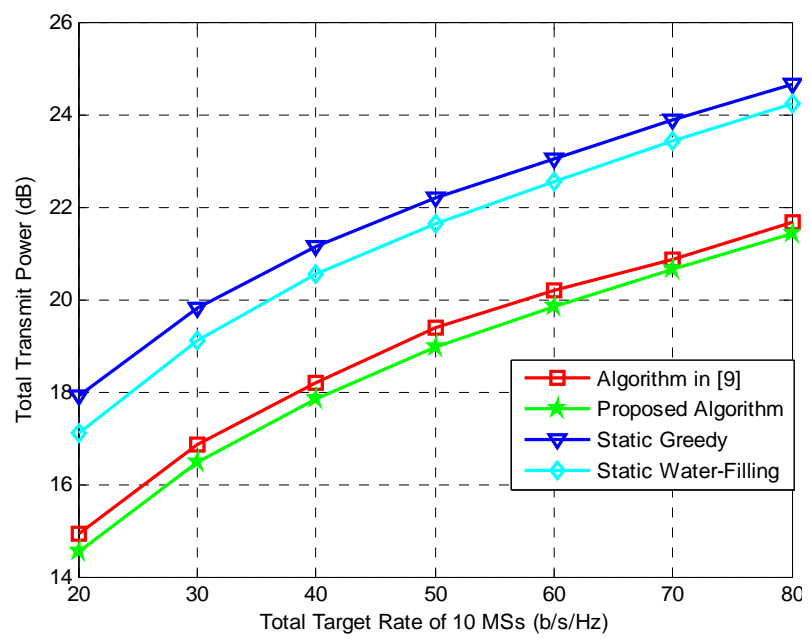

Figure 3. Total transmit power in different total target rate of 10 MSs.

\section{Conclusions}

In this paper, we propose a power-minimizing algorithm in multiuser cooperative relay communication, which fully embodies the concept of green communication and makes a contribution to sustainable development. Simulation results show that compared with other algorithms, the proposed algorithm performs better in terms of the total transmit power consumption while meeting the target rates of users.

\section{Acknowledgements}

This work was supported in part by the National Science Foundation of China (61071119), the Ningbo Natural Science Foundation (2012A610017), the Innovation Team of Ningbo (2011B81002), the Ningbo Natural Science Foundation (2012A610061).

\section{REFERENCES}

[1] W. Shim, Y. Han and S. Kim, "Fairness-Aware Resource Allocation in a Cooperative OFDMA Uplink System," IEEE Transactions on Vehicular Technology, Vol. 59, No. 2, 2010, pp. 932-939.doi:10.1109/TVT.2009.2037328

[2] Z. Shen, J. G. Andrews and B. L. Evans, “Adaptive Resource Allocation in Multiuser OFDM Systems with Proportional Rate Constraints," IEEE Transactions on Wireless Communications, Vol. 4, No. 6, 2005, pp. 2726-2737. doi:10.1109/TWC.2005.858010

[3] H. X. Li, H. W. Luo, X. B. Wang and C. S. Li, "Throughput Maximization for OFDMA Cooperative Relay Networks with Fair Subchannel Allocation,” Wireless Communications and Networking Conference, Budapest, 5-8 April 2009, pp. 1-6.

[4] D. Kivanc, G. Q. Li and H. Liu, “Computationally Efficient Bandwidth Allocation and Power Control for OFDMA,” IEEE Transactions on Wireless Communications, Vol. 2, No. 6, 2003, pp. 1150-1158. doi:10.1109/TWC.2003.819016

[5] N. UL Hassan and M. Assaad, "Low Complexity Margin Adaptive Resource Allocation in Downlink MIMO-OFDMA System," IEEE Transactions on Wireless Communications, Vol. 8, No. 7, 2009, pp. 3365-3371. doi:10.1109/TWC.2009.080210

[6] Y.-B. Lin, T.-H. Chiu and Y. T. Su, "Optimal and Near-Optimal Resource Allocation Algorithms for OFDMA Networks," IEEE Transactions on Wireless Communications, Vol. 8, No. 8, 2009, pp. 4066-4077. doi:10.1109/TWC.2009.080221

[7] W. P. Tam, T. M. Lok and T. F. Wong, "Power-Minimizing Rate Allocation in Cooperative Uplink Systems,” IEEE Transactions on Vehicular Technology, Vol. 58, No. 9, 2009, pp. 4919-4929. 


\section{doi:10.1109/TVT.2009.2027330}

[8] S. Khakurel, L. Musavian and T. Le-Ngoc, "Energy-Efficient Resource and Power Allocation for Uplink Multi-User OFDM Systems," IEEE 23rd International Symposium on Personal Indoor and Mobile Radio Communications, Sydney, 9-12 September 2012, pp. 357-361.
[9] H. Banizaman, S. M. T. Almodarresi and A. A. Tadaion, "Joint Subchannel-Relay Assignment and Bit Allocation for Multi-user Cooperative OFDMA Systems Based on Fairness,” The 18th Iranian Conference on Electrical Engineering, Isfahan, 11-13 May 2010, pp. 276-281. 